\title{
Eine leuchtende Spur
}

\section{Zum Tod des Frankfurter Juristen und Völkerrechtshistorikers \\ Michael Stolleis (20.Juli 1941-18. März 2021)}

\author{
Miloš Vec \\ Professor für Europäische Rechts- und Verfassungsgeschichte, \\ Universität Wien, Wien, Österreich \\ Mitglied des Academic Advisory Board des Journal of the History \\ of International Law \\ milos.vec@univie.ac.at
}

Vielleicht ist es keine unpassende Idee, ein Porträt über den verstorbenen Pionier der globalen Völkerrechtsgeschichte und emeritus editor dieser Zeitschrift Michael Stolleis in einem Münchner Mansardenzimmer beginnen zu lassen. Reisen wir also einige Jahrzehnte zurück, in das München der späten 196oer Jahre, wo der Doktorand und spätere Habilitand Stolleis entscheidende Impulse für seinen Denkstil empfing.

Die Geschichte über den einflussreichen Autor und Lehrer des öffentlichen Rechts würde damit bei seinem eigenen Lehrer beginnen. Michael Stolleis hat oft, gerne und in liebevoller Bewunderung über seine maßgebliche Prägung bei Sten Gagnér berichtet. Und es lohnte und lohnt sich, ihm zuzuhören, wenn Stolleis dessen Charaktereigenschaften Jahrzehnte später pries: „Seine größten Gaben hatte er wohl als Lehrer und als Vorbild. Er hat seine Schüler geliebt, er begleitete sie ein Leben lang, freute und sorgte sich mit ihnen. Und sie wiederum fühlten sich nicht eigentlich als 'Schule', aber verbunden durch diesen ganz ungewöhnlichen, unakademischen Menschen." War er, Stolleis, das nicht auch selbst?

Michael Stolleis wurde am 20. Juli 1941 in Ludwigshafen geboren, wuchs in Gimmeldingen auf, machte eine Lehre zum Winzergehilfen, studierte in Heidelberg und Würzburg Rechtswissenschaften, aber auch Germanistik und Kunstgeschichte. 1965 kam er in der bayrischen Hauptstadt an, lernte im

An English version of this text, translated by Raphael Schäfer, appeared in Vol. 23.3 Journal of the History of International Law and is online available here: https://doi .org/10.1163/15718050-12340188. 


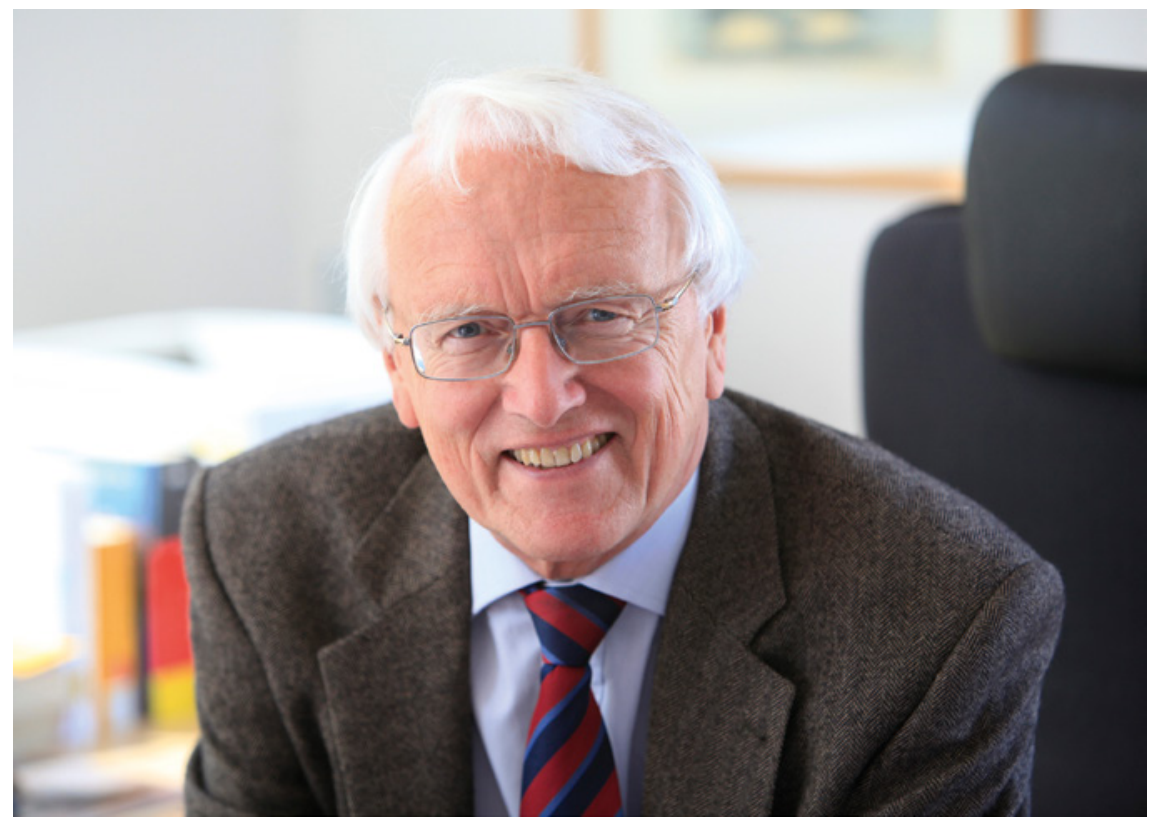

Michael Stolleis 2010 in seinem MPI-Büro

FOTO: SANDRA HAUER/NAH DRAN FOTOGRAFIE

folgenden Jahr Karen kennen, und bezog mit ihr später im Stadtviertel Schwabing in der Schellingstraße eine schöne Wohnung, übrigens in unmittelbarer Nähe zu einem von ihm geschätzten Antiquariat. In München fand er in dem schwedischen Rechtshistoriker Gagnér einen ungewöhnlichen Lehrer, den man sich heute nicht mehr an einer rechtswissenschaftlichen Fakultät und schon gar nicht an einer deutschen vorstellen kann. Gagnér war frisch als Professor für deutsche und nordische Rechtsgeschichte sowie Bürgerliches Recht nach München berufen worden, und Michael Stolleis wurde sein erster Doktorand. Die Impulse, die er von ihm empfing, deuten auf einen weiten europäischen Horizont als Forscher, auf Neugier und kriminalistische Genauigkeit im Umgang mit Quellen (Gagnér war zuvor auch bei der schwedischen Kriminalpolizei tätig gewesen) - und auf die Vorurteilslosigkeit bei der Annäherung an historische Sachverhalte. All diese Tugenden hat Michael Stolleis nach dem Ende seiner Münchner Jahre seinerseits als Universitätslehrer an der Universität Frankfurt (1974-20o6) und später als Direktor des Max-PlanckInstituts für europäische Rechtsgeschichte (1991-2009) an Generationen von zahllosen Doktoranden, Habilitanden, Schülerinnen und Schülern und Gästen aus aller Welt mit Geduld und Wohlwollen, aber auch sanftem Nachdruck vermittelt. 
Meine folgende Erzählung über Michael Stolleis könnte auch ganz anders geschrieben sein. Denn Michael Stolleis war nicht nur als Rechtshistoriker ein Wegbereiter der modernen Völkerrechtsgeschichte, sondern auch sonst ein überaus renommierter Öffentlichrechtler. Sein Wirken mag von anderen Weggefährten und späteren Forscherinnen ganz anders aus ihrer Warte akzentuiert werden, denn viele Menschen fühlten sich von ihm gesehen, gefördert und blieben ihm verbunden. Tatsächlich war der junge Frankfurter Professor Stolleis gerade am Kirchenrecht und am Sozialrecht lebhaft interessiert, und es wurde ihm sogar die Leitung des Münchner Max-Planck-Instituts für Sozialrecht angeboten, die er aber zugunsten der europäischen Rechtsgeschichte, also dem Frankfurter MPI, ausschlug. Auch das Interesse an konfessionellen Prägungen von Recht, Rechtswissenschaft und Gesellschaft begleitete ihn sein Leben lang.

Denn Michael Stolleis war geistig unkonventionell. Er interessierte sich für viel mehr Rechtsfragen und Außerjuristisches, als man glauben konnte. Sein Publikationsverzeichnis spricht Bände über die stupende wissenschaftliche Neugier schon des jungen Mannes. Er las nicht nur fleißig, sondern rezensierte und publizierte Jahr für Jahr in hoher Dichte, qualitätsvoll und immer höchst lesbar. Die Völkerrechtsgeschichte, für die Michael Stolleis zu einem großen und jedenfalls in Deutschland einzigartigen Anreger wurde, war bei ihm eingebettet in eine unermüdliche und unerschrockene Neugier nach der Verbindung von Recht, Geschichte, Politik und auch Moral. Seine Wissenschaftsgeschichte war nie reine Personen-, Institutionen- oder gar juristische Dogmengeschichte. Die Erzählungen, die er bereits in Dissertation und Habilitationsschrift entfaltete, waren in ihren Lehren keineswegs vergleichbar. Die so entstandenen Bücher beschäftigten sich mit zwei ganz verschiedenen Feldern: „Staatsraison, Recht und Moral in philosophischen Texten des späten 18. Jahrhunderts“ erkundete die Spätaufklärung; "Gemeinwohlformeln im nationalsozialistischen Recht" (1974) behandelte als Münchner juristische Habilitationsschrift ein noch junges, viel düstereres und erinnerungspolitisch konfliktreiches Forschungsfeld der Rechtsgeschichte. Denn kaum drei Jahrzehnte nach dem Ende des Zweiten Weltkriegs und der NS-Herrschaft war dies in Deutschland historisch und juristisch ein Minenfeld, über das sich wissenschaftsgeschichtlich zumal an den Rechtsfakultäten - lange Zeit bleiernes Schweigen gelegt hatte. Es würde ihn noch oft beschäftigen; auch familiär veranlasst, denn sein Vater war nicht nur Winzer und Rechtsanwalt, sondern während der Ns-Zeit auch Oberbürgermeister von Ludwigshafen. Liest man heute die Überschriften von journalistischen Interventionen, Essays und Kalenderblättern, fasziniert ihr sachlicher, jedoch gewählter Ton: „Verbrannte Staatsschiffe. Vor hundert Jahren wurde der Jurist und Verfassungshistoriker Ernst Rudolf Huber geboren“ 
und „Durch soviel Türen geschritten. Die Tapeten wechseln, das Recht gilt immer: Vor hundert Jahren wurde Theodor Maunz geboren“. Diese Titel waren bildreich und sprachmächtig, aber nicht polemisch oder verurteilend - sogar, wenn er über Gelehrte wie Maunz schrieb. Dessen Tätigkeit auch nach 1945 als heimlicher Autor und Berater der rechtsextremen „National-Zeitung“ war ein veritabler Skandal des Jahres 1993. Stolleis benannte Dinge, aber er suchte keine billigen Schlagzeilen; Konfrontationen nahm er in Kauf, wenn er Dinge als falsch erkannt hatte; denn er konnte auch sehr moralistisch sein.

Dass Michael Stolleis sich in die NS-Rechtsgeschichte hineinwagte und damit auch eine Universitätskarriere beginnen konnte, war neben seinem offenkundigen Mut einer Reihe von Faktoren geschuldet. Zu nennen ist an erster Stelle sein vollendetes wissenschaftliches Ethos, das solche Werke handwerklich nicht angreifbar machte. Hinzu kam auch bei allen möglichen späteren öffentlichen Ämtern und bei nicht-öffentlichen erinnerungspolitischen Engagements die große charakterliche Integrität des Gelehrten, die politische, institutionelle und persönliche Widerstände milderte. Er war in seiner Sachlichkeit eine unbestechliche Besetzung als Gutachter und für Kommissionen. Er forderte nie etwas für sich persönlich, war in der gemeinsamen Sache verbindlich, diskret und unermüdlich. So hörte ich es von Frankfurter Freunden über sein Engagement für das Fritz Bauer-Institut und für das Wollheim Memorial - jenes bewegende Denkmal für den Ns-Zwangsarbeiter Norbert Wollheim, der seine Entschädigung mühsam, aber schließlich erfolgreich vor deutschen Gerichten in einem Musterprozess erstritt, von dem auch weitere Opfer unmittelbar profitierten. Das Wollheim Memorial steht heute direkt beim elegant-modernen Hauptgebäude auf dem Campus Westend der Goethe Universität, und dieses Hauptgebäude war einst die Hauptverwaltung des IG-Farben-Konzerns gewesen, als Täterin verstrickt in Ns-Gräueltaten und gewissenlos von ihnen profitierend.

Die Goethe-Universität hatte Michael Stolleis als Professor für öffentliches Recht berufen, seinerzeit noch ans Juridicum auf dem Campus Bockenheim. Von seinem Zimmer im 9. Stock aus sah man die Frankfurter Hochhäuser wachsen und die Bewegungen über dem Rhein-Main-Flughafen. Daneben lag das Senckenberg Museum mit seiner weltberühmten naturkundlichen Sammlung und mit dem Physikalischen Verein. Hinter der Sternwarte dominierte der noch viel höhere AfE-Turm, den die Sozialwissenschaften bewohnten. Vor einigen Jahren wurde er gesprengt, jetzt wachsen an der Senckenberganlage Wohntürme mit teuren Eigentumswohnungen bis hin zur Messe. Bockenheim vermisst umso leidenschaftlicher die Studierenden der Geisteswissenschaften, die mit der Universität ins Westend abwanderten. Michael Stolleis blieb der Goethe-Universität über Jahrzehnte hinweg und trotz auswärtiger Rufe treu. 
Er kam in ein von Kriegszerstörungen immer noch gezeichnetes (die Alte Oper eine Ruine), aber umtriebiges Frankfurt, dessen 1914 gegründete „Bürgeruniversität" vielleicht gerade wegen ihrer fehlenden Altehrwürdigkeit offen, liberal und aufklärerisch ausgerichtet war. Stolleis wurde Teil einer kritischen Frankfurter Schule und prägte diese mit. Es schlossen sich lebenslange Freundschaften nicht nur zu seinen Kollegen vom öffentlichen Recht (Erhard Denninger, Ilse Staff) und dem Strafrecht (Wolfgang Naucke, Winfried Hassemer, Klaus Lüderssen), sondern bald auch zur universitär und außeruniversitär weltweit einzigartig starken Rechtsgeschichte. Manche außenstehenden Beobachter sprachen gar von einer „Frankfurter Eintracht“. Besonders enge Bindungen auch persönlicher Art bestanden jedenfalls zu den Rechtshistoriker-Kollegen Bernhard Diestelkamp, Dieter Grimm und Heinz Mohnhaupt; ferner Gerhard Dilcher und seit gemeinsamen Münchener Tagen zu Joachim Rückert. Bis heute ist Frankfurt eine Weltmarke in der Rechtsgeschichte geblieben. Es hat viel mit dem beharrlichen Wirken von Michael Stolleis zu tun.

Im Jahr 1988 erschien der erste Band der Geschichte des öffentlichen Rechts, und man kann aus diesem Meisterwerk viel über Stolleis' Zugang zur Völkerrechtsgeschichte lernen: Denn die dichten Abschnitte über das „Ius Gentium“ waren eingebettet in eine monumentale Erzählung über „Reichspublizistik und Policeywissenschaft", die vielfach antike und mittelalterliche Wurzeln benannte. Sie stand mit der von protestantischen Neuaristotelismus geprägten Politikwissenschaft ebenso in Verbindung wie mit den Themen von Konfessionalisierung, Westfälischem Frieden, Naturrecht und der Guten Policey. Auf dem farbigen Schutzumschlag des Buches symbolisierte ein kosmologisches Bild, ein Kupferstich aus Jacob Bernhard Multz, Repraesentatio Maiestatis, 169o, die Vorstellung einer etatistisch-monarchischen Hoheitsgewalt: Aus dem solaren Zentrum strahlten einzelne Hoheitsrechte, darunter Pax, Bellum und Foedera, als majestätische Sterne im großen Kosmos der juristischen Dinge.

Vielleicht hat mich dieser Band bis an den heutigen Tag deswegen am meisten von allen vieren beeindruckt, weil er so viele verschiedene Fäden zusammenführte, ein „barocker Eisberg der Gelehrsamkeit“. Natürlich war es eine vielfach sehr deutsche Geschichte, es gab ein „Deutsches Völkerrecht", das nicht nur nach außen, sondern auch nach innen galt. Denn das Alte Reich war territorial zersplittert und in seinem Föderalismus zugleich eingebettet in Europa, politisch voller Dispute, aber das öffentliche Recht wurde zu einer gemeinsamen Sprache, in der die Konflikte verhandelt, teilweise gelöst, teilweise dauerhaft ausgeklammert wurden.

1988 war auch das Jahr, in dem Michael Stolleis mein Lehrer im öffentlichen Recht wurde. Er hatte in der Vorlesung Verwaltungsrecht gesagt, dass man ihn wegen Gutachten für Auslandsaufenthalte fragen könnte und er erledigte es 
mit einem mich beschämenden Wohlwollen. Heute weiß ich, dass zahllose Menschen dachten, dass Begegnungen mit ihm einen Unterschied in ihrem Leben ausmachen konnten. Im Gespräch lächelte er gerne. Die Förderungen, die Zugewandtheit und der Zuspruch hörten nicht auf. Er besaß einen "menschenfreundlichen Ton" (um seinen Buchtitel aufzunehmen, unter dem er über die von ihm geschätzten Kalendergeschichten von Johann Peter Hebel witzig nachdachte). Immer noch und vielleicht immer mehr konnte man von ihm lernen. Je älter wir wurden, je mehr wir verstanden, je mehr konkrete Fragen wir hatten, die wir ihm jederzeit stellen durften, desto wertvoller wurde er uns.

Jahrzehntelang hielt Stolleis ein Oberseminar. Er hörte uns zu, immer auf Augenhöhe, folgte den Präsentationen nachkommender Generationen aufmerksam, wechselte dabei seine Brillen, machte Einträge in seinen schwarzen Kalender-Büchern, die er aus seiner erstaunlich leeren Aktentasche zog, und sprach am Ende immer Ermutigungen, aber manchmal auch einen mahnenden Rat aus. Er redete oft leise. Der gemäßigte Ton der väterlichen Strenge speiste sich aus Fürsorglichkeit. Denn er wusste, dass auch fleißige und kluge Leute sich verlieren konnten, dass am Ende nur geschriebene Texte begutachtet, benotet und mit Titeln bedacht werden konnten. Handwerkliches war immer wichtig, Fleiß unabdingbar, und Genialität allein keineswegs hinreichend für eine seriöse Arbeit.

Es fiel umso leichter, diese Maßstäbe und Kritik von ihm anzunehmen, weil Stolleis' Anforderungen auch immer für ihn selbst galten. Konsequent präsentierte er auch seine eigenen Forschungen in diesem Rahmen und stellte sie uns Jüngeren zur Diskussion. Er war Zeit seines Lebens - und erstaunlicherweise selbst auf dem Zenit seiner Verpflichtungen als Wissenschaftsmanager - nie ein reiner Organisator von Forschung. Allen, die ihn von Ferne und Nähe beobachten durften, war und blieb es ein Rätsel, wie er parallel zur Leitung des Max-Planck-Instituts und eingebunden in zahllose andere Projekte sein 1988 begonnenes monumentales Werk der "Geschichte des öffentlichen Rechts in Deutschland“, mit den weiteren Bänden 1992 (unter Auslassung des Völkerrechts) und 1999 fortsetzte und 2012 (dann bereits als Emeritus) zu einem international aufsehenerregenden Abschluss brachte. Übersetzungen dieses und vieler anderer Werke (etwa des „Auge des Gesetzes“) in zahlreiche Sprachen folgten. Allen, die ihn kannten, blieb es ein Rätsel, wie man so viel lesen, wissen und schreiben konnte. Er war ein bemerkenswerter Mann und die Ikone eines Forschers. Für all das wurde er mit wichtigsten nationalen und internationalen Preisen und Auszeichnungen bedacht. Nicht zuletzt wurde er für seine Verdienste als Forscher und öffentliche moralische Autorität im Jahr 2015 mit dem Großen Bundesverdienstkreuz mit Stern gewürdigt. Aber wichtiger waren ihm wohl die Freundschaften, die er in aller Welt schloss. 
Immer wieder und bis in die jüngste Vergangenheit hat Michael Stolleis seine nicht alleine auf das Juristische beschränkte Perspektive auf die normativen Verhältnisse zwischen autonomen politischen Akteuren beibehalten und über sie publiziert. „Der Streit um den Vorrang, oder: Der Wasunger Krieg“ schildert einen typisch frühneuzeitlichen Zeremoniellstreit zwischen Territorien. Er führt zu militärischen Auseinandersetzungen auf Duodez-Niveau, aber es gab schließlich eine schiedsgerichtliche Lösung. Stolleis findet darin „Elemente von Komik und Theatralik" und spendet dem Reichskammergericht ein

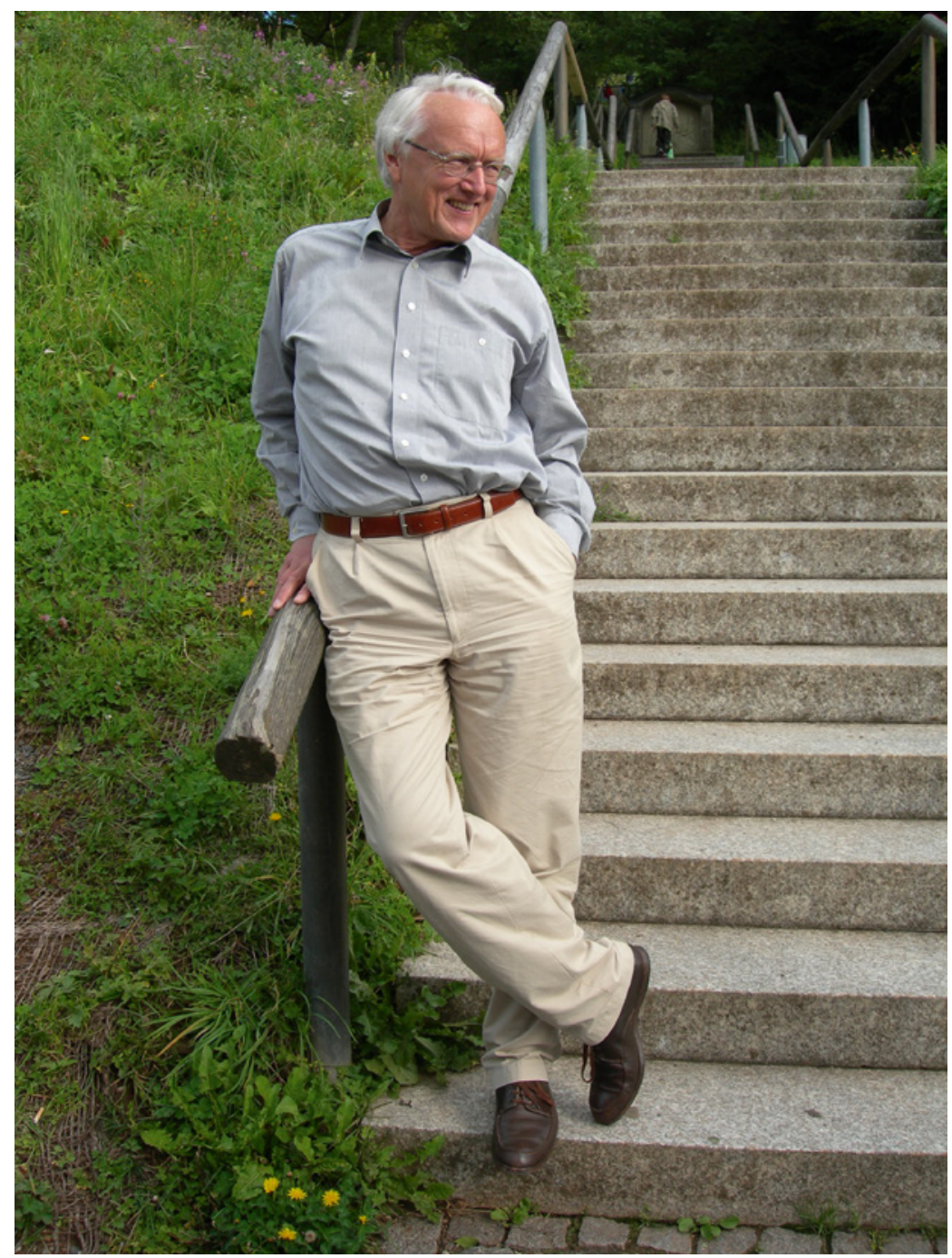

Michael Stolleis auf dem Betriebsausflug des MPI 2006 in der Rhön FOTO: MILOŠ VEC 
Lob für seine schnelle, friedewahrende Arbeit. Im Fazit erscheint ein Muster von Konfliktlösung oder jedenfalls Kontrolle über potenziell gewaltsame Akteure mit einer „Kombination von diplomatischen Aktionen und militärischen Drohungen“. „Die Prinzessin als Braut“ erzählt vormodernes Eherecht mit völkerrechtlichem Einschlag, nämlich die Verheiratung der Prinzen und Prinzessinnen als „Staatsaktion von höchster Bedeutung“; wieder wird die Rolle von „Reichsrecht und vom Völkerrecht“ betont. Und „Erzählen“ meint im wörtlichen Sinne einen Ton, der Anschaulichkeit, Lebendigkeit und Klarheit in Geschichten erreichte.

Er wurde zum 1.11.1991 Co-Direktor von Dieter Simon im neuen Gebäude an der Nidda (im Oktober 199o bezogen) und brachte dem außeruniversitären Institut der Max-Planck-Gesellschaft und dem Fach „europäische Rechtsgeschichte“ zahlreiche neue Forschungsfelder: „Gute Policey“, Völkerrechtsgeschichte, Rechtsgeschichte Südosteuropas und Geschichte des Technikrechts hatte es in dieser Form bisher noch nicht gegeben. Das Max-Planck-Institut eröffnete Michael Stolleis Möglichkeiten zur Erforschung der Rechtsgeschichte, die die perspektivischen und thematischen Beschränkungen des universitären Fachs ebenso überwand wie performativ kritisierte. Denn Stolleis forderte nicht nur Öffnungen, Erweiterungen, Aufbrüche. Er schrieb nicht nur vielfach über die Methode der Rechtsgeschichte, dachte dabei insbesondere über ihre Beziehung zur Literatur nach, räsonierte über die „Entbehrlichkeit von ,Begriff' und ,Tatsache“" in der Historiographie. Nein, er schritt selbst voran und reformierte das Fach Rechtsgeschichte in Person oder stellte entscheidende Weichen. Für die Juristische Zeitgeschichte installierte er mithilfe der VolkswagenStiftung einen neuen Lehrstuhl an der Frankfurter Universität, der sehr qualifiziert mit Joachim Rückert besetzt wurde, ebenso Gagnér-Schüler. Ein weiteres dieser Pionierprojekte war die von der DFG finanzierte Gruppe „Deutsche Völkerrechtswissenschaft im Europa des 19. und 20. Jahrhunderts“. Sie bestand von 1997 bis 2001 und war ein international einzigartiger Cluster von NachwuchsforscherInnen zur Völkerrechtsgeschichte, aus dem zahllose, qualitätsvolle Dissertationen hervorgingen. 2001 gründete er die seither im Nomos-Verlag erscheinenden "Studien zur Geschichte des Völkerrechts“ als Buchreihe, 2004 wurde er Mitherausgeber dieser Zeitschrift, des Journal of the History of International Law.

Dieser Aufbruch, nicht von ungefähr unternommen in einem außeruniversitären Forschungsinstitut, koinzidierte mit einer internationalen Hinwendung zur Völkerrechtsgeschichte. Michael Stolleis rezensierte im Juli 2002 prominent und ausführlich Martti Koskenniemis „Gentle Civilizer“ in der FAZ und unterstützte diesen zunehmend global sichtbaren „Turn to History“ tatkräftig: „Koskenniemis Buch ist eine große Leistung europäischer Wissenschaftsgeschichte 
und ein literarisches Meisterwerk" hieß es im Schlusssatz der würdigenden Besprechung. Zugleich konnte man aber immer wieder den ungehaltenen und mit dem Zustand des Fachs unzufriedenen Stolleis vernehmlich hören, der gerne auch in dieser Hinsicht mehr Innovation einforderte: „Aber das [Erscheinen des Buchs von Koskenniemi, Mv] verbessert eigentlich nicht die Lage der Völkerrechtsgeschichte in Deutschland selbst. Das Fach ist praktisch nicht existent“, schrieb er 2006. Mit Masaharu Yanagihara gab er 2004 den Band „East Asian and European Perspectives on International Law" heraus. Das von beiden gezeichnete Vorwort wirft gerade heute aktuelle Forschungsfragen auf:

It is also arguable whether modern European international law was universal in the world in the latter half of the 19th century. We should realize that there are two aspects to the question. The first one is whether international law was monolithic in Europe, or whether each European country had its own international law such as German international law, or English international law. The second one is whether modern European international law could be actually applied universally in the world.

Weil Stolleis ein unfassbar informierter und gebildeter Gelehrter war, blieb seine Völkerrechtsgeschichte immer eingebettet in größere geschichtliche und geographische Zusammenhänge. Alles Recht war bei ihm „Recht im Kontext“. Er scheute dabei auch nicht davor zurück den Finger auf wunde Punkte zu legen. Michael Stolleis war bald nach der Wiedervereinigung MPI-Direktor geworden und schrieb kritisch über die Ausgestaltung des „Beitritts“, zudem forschte er auch immer wieder rückblickend zur Geschichte des DDR-Rechts und förderte die Rechtsgeschichte und die Rechtshistoriker in Ostdeutschland. Während seiner Lebensspanne erlebte er die politische und juristische Integration Europas, an dem das erst geteilte und später wiedervereinigte Deutschland mit all seinen Erwartungen, Hoffnungen und seinem historischen Ballast teilhatte. Nationale Staatsrechte, Europarecht und Völkerrecht musste man angemessener Weise in ihren wechselseitigen Verflechtungen begreifen. Auch Nationalismus und Internationalismus waren aufeinander bezogen. Man konnte die eine Idee nicht ohne die andere verstehen. Mit Blick auf die Rechtsvergleichung im öffentlichen Recht des späten 19. Jahrhunderts notierte er 1998: „Wachsender Nationalismus und Internationalismus erwiesen sich also nicht etwa als Gegensätze, sondern gewissermaßen als siamesische Zwillinge. Je mit mehr man sich näher rückte, desto schärfer wurden die nationalistischen Abgrenzungsphrasen. Je stärker die internationale Vernetzung wurde, desto mehr wuchs auch das Bedürfnis nach Wahrung der nationalen Identität." 
Die Entstehung und Ausbreitung von Recht mussten im Zusammenhang mit ihren Gegenbegriffen und gegenläufigen Entwicklungen gesehen werden: dem Unrecht in Rechtsform und dem Vergehen von Recht. Zeit seines Lebens blieb er dem Thema des nationalsozialistischen Unrechtsstaats nicht nur intellektuell als Forscher verbunden, der klug über nationalsozialistisches Völkerrecht schrieb, sondern auch als aktiver Teil der (Frankfurter und deutschen) Bürgergesellschaft/Zivilgesellschaft. Denn die Ns-Verbrechen hatten nicht nur Spuren in der Wissenschaftsgeschichte hinterlassen, sondern auch neue Normen generiert, und immer wieder verhandelten Zivilgesellschaften neu, wie sie ihre eigene Vergangenheit sahen, bewerteten und juristisch fassen wollten. "Crimes against Humanity“ und "Genocide“ lauteten die juristischmoralischen Anklagen in tatbestandlicher Form, in denen Menschenrechte und Völkerrecht zusammenkamen. Sie erlebten nach 1945 im Völkerstrafrecht sehr wechselhafte Konjunkturen. Michael Stolleis war bewusst, dass „Erwartungen an das Recht" ein Maßstab der Wahrnehmung und auch möglicher Kritik von Entwicklungen waren - und gerade auch Rechtsstaaten bzw. die Weltstaatengemeinschaft überfordern konnten. Er formulierte selbst freimütig "Juristenträume" nach rechtspolitischen und curricularen Reformen, gerade wenn diese sich tagespolitisch nicht durchsetzen ließen. Seine Erwartungen an die Rechtsgeschichte waren ein offenes und unabgeschlossenes Projekt. Er forderte das Fach und seine Vertreter, die Nachbarwissenschaften und die Öffentlichkeit immer wieder heraus, aber nicht aus einer bequemen Kritikerrolle, sondern als Teil der Welle, die wir selbst sind.

Seine Bücher richteten sich keineswegs allein an ein juristisches Fachpublikum, sondern wie seine anlässlich des 6o-jährigen Jubiläums des Bundesverfassungsgerichts herausgegebene Festschrift „Herzkammern der Republik“ an eine allgemeine Öffentlichkeit. Er trug maßgeblich dazu bei, die Bedeutung des Rechts in öffentlichen Diskursen prominent zu platzieren. Alleine für die FAZ verfasste er rund 140 Artikel. Der letzte erschien am Freitag, den 12. Februar 2021 und war eine berührende Buchbesprechung: „Ein Junge in Auschwitz. Singuläres Zeugnis: Tomás Radils Erinnerungen“.

Im Haus der Familie Stolleis in Kronberg am Taunus steht nahe beim Eingangsbereich ein schönes, weißes, perfekt in die Wand eingefügtes Regal (ein Geschenk seiner Frau Karen) mit alten ausgewählten Leder- und Pergamentbänden. Reichshistorie, Ius Publicum, Verfassungsrecht des 19. Jahrhunderts und anderes sind dort in verdichteter Form beiläufige Zeugen seines Schaffens. Ich vermute dort Johann Stephan Pütters dreibändige „Litteratur des Teutschen Staatsrechts" und bin sicher, dass jedenfalls Robert von Mohl darunter ist, dem er in gewisser Weise rund 150 Jahre später nachfolgte, mit seinerseits 


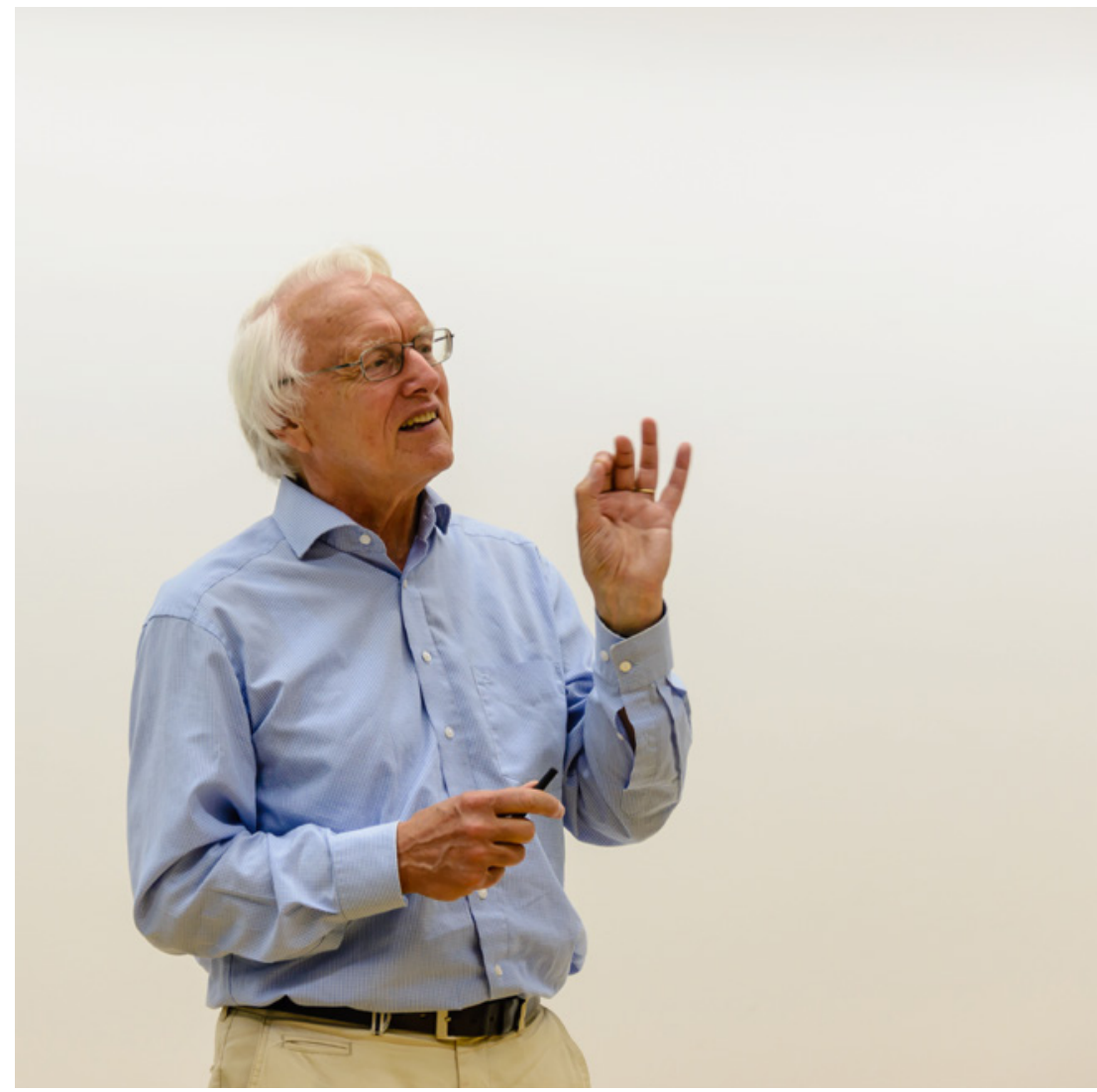

Michael Stolleis bei der Summer Academy des MPI am 22. Juli 2016, kurz nach seinem 75. Geburtstag

FOTO: CHRISTIANE BIRR

vollendeter Übersicht über die „Geschichte und Literatur der Staatswissenschaften“, verfasst mit Disziplin und formuliert mit Augenmaß in den Urteilen. Stolleis' kleine Sammlung ist kein Vergleich zur legendären Bibliothek von Sten Gagnér, die „eine Arche Noah der Gelehrsamkeit“ war und 9676 Bände zählte (die rund 25 Fachzeitschriften, die er fortlaufend bezog, nicht mitgerechnet). Aber aus Besitztümern und erst recht Luxus machte Michael Stolleis sich nichts und verblüffte oft mit seiner Bescheidenheit und in seinem Verzicht. Noch Jahrzehnte später erinnern sich seine Weggefährten, dass der immer jugendlich wirkende Professor zu Besprechungen mit Frankfurter Studierenden in Jeans erschien. Er besaß einen jungenhaften Charme. Er war eigensinnig, ungeduldig und ging gerne alleine voran. Von Gesellschaften 
entfernte er sich oft früh. In seinen freien Stunden töpferte er liebevoll und mit sehr vorzeigbaren Ergebnissen. Gerne machte er Wanderungen und Radtouren mit Freunden, teils mehrtägig. Wenn seine Kinder Friederike und Peter am Ende des Wanderns ermüdeten, erzählte er ihnen aus der Odyssee. Vor kaum mehr als einem Jahr spazierte ich mit ihm durch den Taunus, wir begutachteten Orkanschäden im hohen Nadelwald und ließen uns durch gesperrte Wege nicht beschränken. Er begeisterte sich für Kunst und Literatur, und sein Schreiben profitierte unverkennbar davon. Er, der Unprätentiöse, war ein Ästhet und Stilist in jedem Wort und jedem Satz. Als sein Lehrer Sten Gagnér im Jahr 2000 starb, widmete Stolleis ihm in der FAz einen liebevollen Nachruf und ließ einen programmatischen ersten Absatz mit der Würdigung seiner Persönlichkeit enden: „Seine Begabung zu Freundschaften war legendär. Wo er auftauchte, entfaltete sich eine ansteckende Fröhlichkeit, zugleich aber ein intensiver wissenschaftlicher Austausch." Die Überschrift, ein einziges Wort: „Gewährenlassen“. Michael Stolleis ist am 18. März 2021 mit 79 Jahren, im gleichen Alter wie sein Lehrer Sten Gagnér, gestorben. Am Ende seines Nachrufs auf jenen standen drei Worte, und auch auf den Verfasser Michael Stolleis passen sie: „Eine leuchtende Spur.“

\section{Selected References}

Staatsraison, Recht und Moral in philosophischen Texten des späten 18. Jahrhunderts (Meisenheim: Anton Hain, 1972).

Gemeinwohlformeln im nationalsozialistischen Recht (München: J. Schweitzer, 1974).

'Aufgaben der Neueren Rechtsgeschichte, oder: hic sunt leones'. Rechtshistorisches Journal 4 (1985), 251-264.

Geschichte des öffentlichen Rechts in Deutschland, Bd. I: Reichspublizistik und Policeywissenschaft 1600-1800 (München: C. H. Beck, 1988).

'Angst (West)'. Frankfurter Allgemeine Zeitung (26. Mai 199o), B2 [Bilder und Zeiten].

Geschichte des öffentlichen Rechts in Deutschland, Bd. II: Staatsrechtslehre und Verwaltungswissenschaft 1800-1914 (München: C. H. Beck, 1992).

'Eckstein des Anstoßes. Theodor Maunz und die Abgründe der "herrschenden Lehre". Frankfurter Allgemeine Zeitung (21. Dezember 1993), 27.

[ed.] Juristische Zeitgeschichte. - Ein neues Fach? (Baden-Baden: Nomos, 1993).

'Gemeineuropäisches Verfassungsrecht - historisch entwickelt?', in Einigung und Zerfall: Deutschland und Europa nach dem Ende des Ost-West-Konflikts, ed. Gerhard Lehmbruch (Opladen: Leske+Budrich, 1995), 149-165.

Das "europäische Haus" und seine Verfassung. Kritische Vierteljahresschrift $78(33)$ (1995), 275-297. 
[ed.] Policey im Europa der Frühen Neuzeit (Frankfurt am Main: Klostermann, 1996). Rechtsgeschichte als Kunstprodukt. Zur Entbehrlichkeit von "Begriff" und "Tatsache" (Baden-Baden: Nomos, 1997).

Nationalität und Internationalität: Zur Entstehung der Rechtsvergleichung des öffentlichen Rechts im 19. Jahrhundert (Stuttgart: Franz Steiner, 1998).

Geschichte des öffentlichen Rechts in Deutschland, Bd. III: Staats- und Verwaltungsrechtswissenschaft in Republik und Diktatur 1914-1945 (München: C. H. Beck, 1999).

'Gewährenlassen. Zum Tod des Rechtshistorikers Sten Gagnér'. Frankfurter Allgemeine Zeitung (26. Mai 2000), 46.

'Sten Gagnér (1921-2000), ein großer Lehrer der europäischen Rechtsgeschichte‘.Quaderni Fiorentini 29(1) (2000), 560-569.

'Die Prinzessin als Braut', in Verfassung - Philosophie -Kirche. Festschrift für Alexander Hollerbach zum 7o. Geburtstag, eds. Joachim Bohnert, Christof Gramm, Urs Kindhäuser, Joachim Lege, Alfred Rinken und Gerhard Robbers (Berlin: Duncker \& Humblot, 2001), 45-57.

'Durch soviel Türen geschritten. Die Tapeten wechseln, das Recht gilt immer: Vor hundert Jahren wurde Theodor Maunz geboren'. Frankfurter Allgemeine Zeitung (1.September 2001), 45 .

'Unterm Vergrößerungsglas. Martti Koskenniemis glänzende Geschichte des Völkerrechts' [Review of: Koskenniemi, Martti. The Gentle Civilizer of Nations. The Rise and Fall of International Law 1870-1960 (Cambridge: Cambridge University Press, 2001]. Frankfurter Allgemeine Zeitung (22. Juli 2002), 37, [Finnische Version in Lakimies 6 (2002) 1029-1032].

'European Legal History - Traditions and Visions', in Rättshistoria i förändring. Olinska stiftelsen 50 år, ed. Kjell Å. Modéer (Stockholm: Roennells, 2002), 167-179.

Reluctance to Glance in the Mirror: The Changing Face of German Jurisprudence after 1933 and post 1945 (Chicago: University of Chicago, 2002).

Der menschenfreundliche Ton. 24 Kalendergeschichten von Johann Peter Hebel mit kleinem Kommentar (Frankfurt am Main: Insel, 2003, 2. Aufl. 2004, 3. Aufl. 2006).

Das Auge des Gesetzes. Geschichte einer Metapher (München: C. H. Beck, 2003).

'Erwartungen an das Recht'. Frankfurter Allgemeine Zeitung (30. Dezember 2003), 7 [vollständiger Fassung in:Jahrbuch der Akademie der Wissenschaften zu Göttingen 2003, 43-59].

'Verbrannte Staatsschiffe. Vor hundert Jahren wurde der Jurist und Verfassungshistoriker Ernst Rudolf Huber geboren'. Frankfurter Allgemeine Zeitung (7. Juni 2003), 36.

[ed. mit Masaharu Yanagihara, eds. East Asian and European Perspectives on International Law (Baden-Baden: Nomos, 2004).

'Rechtlich verfasstes Europa', in Eine Verfassung für ein Europa mit 25 Mitgliedstaaten: Vielfalt und Einheit zugleich, eds. Manfred Zuleeg, Marjolaine Savat und JeanPhilippe Derosier (Baden-Baden: Nomos, 2005) 31-38. 
'The dissolution of the Union between Norway and Sweden within the context of international law - hundred years later', in Rett, Nasjon, Union. Den svensk-norske unionens rettslige historie 1814-1905, eds. Ola Mestad und Dag Michalsen (Oslo: Universitetsforlag, 2005), 35-48.

'Vom Verschwinden verbrauchten Rechts', in Summa. Festschrift Dieter Simon zum 70. Geburtstag, eds. Rainer Maria Kiesow, Regina Ogorek und Spiros Simitis (Frankfurt am Main: V. Klostermann, 2005), 539-558.

'Zur Ideengeschichte des Völkerrechts 1870-1939', in Ideen als gesellschaftliche Gestaltungskraft im Europa der Neuzeit. Beiträge für eine erneuerte Geistesgeschichte, eds. Lutz Raphael und Heinz-Elmar Tenorth (München: Oldenbourg, 20o6), 161-171.

'Vorwort'. Katalog der Bibliothek (1921-2000) von Sten Gagnér, CD-ROM, MPIerR 2007, $2-3$.

'Rechtsgeschichte, Verfassungsgeschichte', in Grundkurs Geschichte, ed. Hans-Jürgen Goertz (Reinbek: Rowohlt, 3. Aufl. 2007), 391-412.

'Against Universalism - German International Law under the Swastika: Some Contributions to the History of Jurisprudence 1933- 1945'. German Yearbook of International Law 50 (2007), 91-110.

Rechtsgeschichte schreiben. Rekonstruktion, Erzählung, Fiktion? (Basel: Schwabe Verlag, 2008).

'Juristenträume'. Merkur 62(12) (2008), 1128-1132.

'Ein Staat ohne Staatsrecht, eine Verwaltung ohne Verwaltungsrecht? - Zum öffentlichen Recht in der Rechtswissenschaft der DDR'. Berlin-Brandenburgische Akademie der Wissenschaften, Berichte und Abhandlungen, Bd. 15 (Berlin: Akademie Verlag, 2009), 39-55.

"Sozialistische Gesetzlichkeit". Staats- und Verwaltungsrechtswissenschaft in der DDR (München: C. H. Beck, 20o9).

'Europa als Rechtsgemeinschaft', in Europa als kulturelle Idee. Symposion für Claudio Magris, ed. Stefan Kadelbach (Baden-Baden: Nomos, 2010), 71-81.

[ed.]. Herzkammern der Republik. Die Deutschen und das Bundesverfassungsgericht (München: C. H. Beck, 2011).

Geschichte des öffentlichen Rechts in Deutschland, Bd. IV: Staats- und Verwaltungsrechtswissenschaft in West und Ost 1945-199o (München: C. H. Beck, 2012).

'Heinrich Bernhard Oppenheim (1819-1880). Rechtsphilosophie und Völkerrecht um 1848', in Naturrecht und Staat in der Neuzeit. Festschrift Diethelm Klippel zum 70. Geburtstag, eds. Jens Eisfeld, Martin Otto, Louis Pahlow und Michael Zwanzger (Tübingen: Mohr Siebeck, 2013), 5०3-518.

'Staatsverträge in der Neueren Staats- und Völkerrechtsgeschichte'. Zeitschrift für Altorientalische und Biblische Rechtsgeschichte 19 (2013), 1-11.

Nahes Unrecht, fernes Recht. Zur Juristischen Zeitgeschichte im 20. Jahrhundert (Göttingen: Wallstein, 2014). 
Margarethe und der Mönch. Rechtsgeschichte in Geschichten (München: C. H. Beck, 2015).

'Methode der Rechtsgeschichte', in Handwörterbuch zur Deutschen Rechtsgeschichte, Bd. III, eds. Albrecht Cordes, Hans-Peter Haferkamp, Heiner Lück, Ruth SchmidtWiegand und Dieter Werkmüller (Berlin: Erich Schmidt Verlag, 2016), Sp. 1475-1483. 'Ein Junge in Auschwitz. Singuläres Zeugnis: Tomás Radils Erinnerungen' [Rezension von Radil, Tomás. Ein bisschen Leben vor diesem Sterben, aus dem Tschechischen von Hubert Laitko (Wuppertal: Arco Verlag, 2020)]. Frankfurter Allgemeine Zeitung (12. Februar 2021), 10. 